\title{
A novel approach to navigated implantation of S-2 alar iliac screws using inertial measurement units
}

\author{
Gregory F. Jost, MD, ${ }^{1}$ Jonas Walti, MSc, ${ }^{2}$ Luigi Mariani, MD, ${ }^{3}$ and Philippe Cattin, $\mathrm{PhD}^{2}$ \\ ${ }^{1}$ Spine Surgery and ${ }^{3}$ Department of Neurosurgery, University Hospital Basel; and ${ }^{2}$ Medical Image Analysis Center, University of
} Basel, Switzerland

\begin{abstract}
OBJECT The authors report on a novel method of intraoperative navigation with inertial measurement units (IMUs) for implantation of S-2 alar iliac (S2Al) screws in sacropelvic fixation of the human spine and its application in cadaveric specimens.

METHODS Screw trajectories were planned on a multiplanar reconstruction of the preoperative CT scan. The pedicle finder and screwdriver were equipped with IMUs to guide the axial and sagittal tilt angles of the planned trajectory, and navigation software was developed. The entry points were chosen according to anatomical landmarks on the exposed spine. After referencing, the sagittal and axial orientation of the pedicle finder and screwdriver were wirelessly monitored on a computer screen and aligned with the preoperatively planned tilt angles to implant the S2AI screws. The technique was performed without any intraoperative imaging. Screw positions were analyzed on postoperative CT scans.

RESULTS Seventeen of 18 screws showed a good S2Al screw trajectory. Compared with the postoperatively measured tilt angles of the S2AI screws, the IMU readings on the screwdriver were within an axial plane deviation of $0^{\circ}$ to $5^{\circ}$ in 15 $(83 \%)$ and $6^{\circ}$ to $10^{\circ}$ in $2(11 \%)$ of the screws and within a sagittal plane deviation of $0^{\circ}$ to $5^{\circ}$ in $15(83 \%)$ and $6^{\circ}$ to $10^{\circ}$ in $3(17 \%)$ of the screws.
\end{abstract}

CONCLUSIONS IMU-based intraoperative navigation may facilitate accurate placement of S2AI screws. http://thejns.org/doi/abs/10.3171/2015.6.SPINE1594

KEY WORDS spine; navigation; S-2 alar iliac screws; image-guided surgery; inertial measurement unit; sacral

$\mathrm{S}$ PINAL instrumentation to the pelvis with S-2 alar iliac (S2AI) screws features excellent pullout resistance, ${ }^{11}$ inline rod placement, and low profile. ${ }^{1} \mathrm{~S} 2 \mathrm{AI}$ screws may be implanted using fluoroscopic guidance ${ }^{13}$ or intraoperative 3D image guidance. ${ }^{12}$ Whereas the ubiquitous fluoroscopic guidance can verify placement of the S2AI screws above the sciatic notch, correct angulation of the S2AI screw in the axial plane depends on the surgeon's estimation and experience and can only be confirmed with a fluoroscopic pelvic inlet view after the S2AI screw has been implanted. ${ }^{8}$ On the other hand, 3D image guidance provides visualization of all planes, enabling the surgeon to maximize both length and purchase of the S2AI screw, ${ }^{12}$ but availability of this technology is still limited. A potential low-cost alternative would be to use inertial measurement units (IMUs) to reproduce the sagittal and axial tilt angles of the S2AI trajectory. IMUs are electronic systems that power motion-sensitive applications in smartphones and tablet computers or switch the screen from portrait to landscape mode. In a previous publication, we described the technical aspects and preliminary data of using IMUs to guide the implantation of pedicle screws and S2AI screws in four cadavers. ${ }^{14}$ In this laboratory study, we have extended our investigation and analysis of using an IMU-equipped pedicle finder and screwdriver to confirm the angulation of the S2AI screw.

\section{Methods}

The study protocol was reviewed and approved by the local institutional review board (Ethikkomission Nordwest- und Zentralschweiz [EKNZ]). Nine human cadavers, aged 62 to 90 years (mean 78 years), were used for the study. Six were male, 3 were female. Fine-cut CT 
scans of the spine and pelvis were performed and viewed in the multiplanar reconstruction mode (MPR) using the research version of the DICOM viewer Osirix (Pixmeo). The sacrum was exposed, and the entry points for the S2AI screws were selected.

\section{Preoperative Planning}

Briefly, the MPR cursor was set at the entry point of the S2AI screw as previously described $1 \mathrm{~mm}$ distal and $1 \mathrm{~mm}$ lateral to the S-1 dorsal foramen, ${ }^{10}$ and the planes were oriented to display the trajectory of the S2AI screws in the axial and sagittal planes. At first, the mediolateral tilt and the trajectory length were recorded (Fig. 1). While the sagittal orientation of the S2AI trajectory was maintained in the MPR mode, the MPR cursor was shifted from the entry point of the S2AI screw to the paramedian sacral plane. Then, the sagittal angular tilt in reference to a plumb line through the sacral plane was recorded (Fig. 2). These steps were repeated for the contralateral side. In the last experiment, the sagittal tilt was referenced to the plumb line through the L5-S1 supraspinous ligament. All angles were entered into the custom-developed software on a MacBook Air running Windows. In the last 2 cadavers, the distance from the ipsilateral L-5 transverse process to the entry point of the S2AI screw was measured for intraoperative reproduction and confirmation with a divider.

\section{Hardware Setup}

The pedicle finder and screwdriver were each equipped with an IMU (MTw sensor, Xsens Technologies B.V., Enschede, The Netherlands) in a custom-made 3D-printed housing (Fig. 3 left). The orientation of the pedicle finder and screwdriver was wirelessly transmitted to the MacBook Air and displayed in digits and on a target, reproducing the axial orientation on the $y$-axis and the sagittal orientation on the x-axis (Figs. 4 and 5 lower).

\section{Intraoperative Guidance}

The lumbosacral area was exposed, and the entry points for the S2AI were decorticated. Entry points were chosen as previously described $1 \mathrm{~mm}$ distal and $1 \mathrm{~mm}$ lateral to the S-1 dorsal foramen. ${ }^{6}$ In the last 2 cadavers, we additionally confirmed the correct position of the entry point by reproducing the preoperatively measured distance to the L-5 transverse process with a divider. After calibration of the sensors, the pedicle finder was first oriented along the imaginary reference line for zeroing of the IMU: in the sagittal plane perpendicular to the paramedian sacral plane and in the axial plane neutrally (without any tilt) (Fig. 4 upper). A custom-made 3D-printed device was used for zeroing the pedicle finder and screwdriver on the last 2 cadavers (Fig. 3 right). In terms of rotation, the sensor box was strictly aligned in the cranio-caudal axis of the spine. While the pedicle finder or screwdriver was held in this position, an assistant pressed a USB foot switch to zero the sensor (Fig. 4 lower). Next, the pedicle finder was moved to the entry point, and the orientation of the tool was controlled on the screen by matching it with the planned sagittal and axial tilt (Fig. 5 lower). This was done by overlapping the blue dot (present orientation) with the green dot (target orientation, Fig. 5 lower). With the pedicle finder advanced by $70 \mathrm{~mm}$, actual IMU readings in the axial and sagittal planes were registered. The pedicle finder was pulled out, and the trajectory was probed and tapped across the S-2 iliac joint. Referencing was repeated with the screwdriver, which was then also oriented to the planned sagittal and axial tilt for implanting the screw (Fig. 6). Before the screw was released, the IMU's displayed sagittal and axial tilt angles were recorded.

\section{Postoperative Analysis}

A postoperative CT scan was performed, and the axial and sagittal tilt angles of the implanted S2AI screws were measured in the MPR mode of Osirix as described above for the trajectory planning. The screw trajectory was checked for cortical bone breaches.

\section{Statistical Evaluation}

The chi-square test was used for group comparison. The significance level was set at $\mathrm{p}=0.05$.

\section{Results}

\section{Accuracy of IMU-Guided S2AI Instrumentation}

The planned mean axial tilt angle was $42^{\circ}$ (range $36^{\circ}-$ $48^{\circ}$ ). With the plumb line through the paramedian sacral plane as reference, the planned sagittal tilt angle was $0^{\circ}$ in 12 S2AI screws. The handle of the pedicle finder and screwdriver had to be tilted $4^{\circ}$ toward the head in 1 screw and $5^{\circ}, 9^{\circ}$, and $13^{\circ}$ toward the feet in another 3 screws. With the plumb line through the L5-S1 supraspinous ligament as reference in the last experiment, the handles of the tools had to be tilted by $20^{\circ}$ and $18^{\circ}$ toward the head. Seventeen of 18 screws showed good S2AI screw trajectories. In relation to the planned tilt angles, a deviation within $5^{\circ}$ was achieved by $13(72 \%)$ in the axial plane and by 8 $(44 \%)$ in the sagittal plane. Although this difference was not significant $(p=0.1)$, the findings showed a trend toward better results for the axial than the sagittal plane. In the axial plane, another 4 screws (22\%) showed a deviation between $6^{\circ}$ and $10^{\circ}$, and in the sagittal plane, a deviation between $6^{\circ}$ and $10^{\circ}$ was measured in $5(28 \%)$ and a deviation between 11 and $15^{\circ}$ in another 5 (28\%). One screw breached into the pelvis past the sacroiliac joint (Fig. 7). It was the second screw in the study and was inserted without prior tapping across the sacroiliac joint, which we identified as the cause of final misplacement. The deviation was clearly indicated by the IMU past the sacroiliac joint, and in relation to the planned axial tilt angle, the final deviation was $18^{\circ}$.

\section{Accuracy of the IMU}

It was of interest to see not only whether it was possible to implant screws as planned, but even more, whether the final IMU readings before releasing the fully implanted screw corresponded to the postoperatively measured angles. Final IMU readings were within an axial plane deviation of $0^{\circ}$ to $5^{\circ}$ in 15 screws $(83 \%)$ and $6^{\circ}$ to $10^{\circ}$ in 2 screws (11\%) and within a sagittal plane deviation of $0^{\circ}$ to $5^{\circ}$ in $15(83 \%)$ and $6^{\circ}$ to $10^{\circ}$ in 3 (17\%) (Fig. 8). 

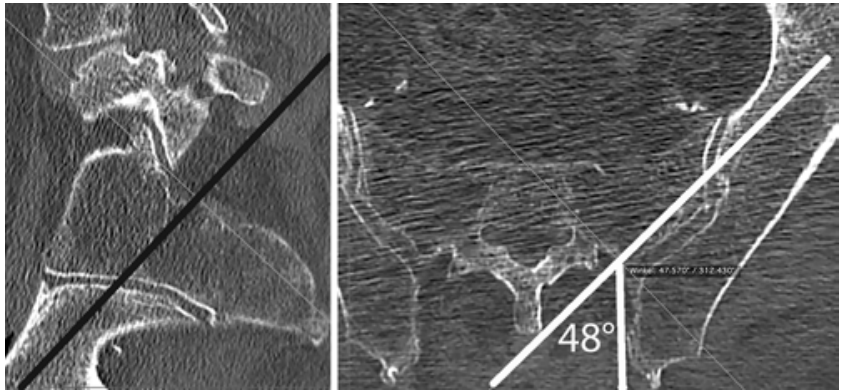

FIG. 1. Multiplanar reconstruction of the preoperative CT scan showing the trajectory for a left S2AI screw (black line on the left panel and white line on the right panel). The mediolateral (axial) tilt as measured on the right panel is $48^{\circ}$.

\section{Timing for Screw Placement}

After exposure and decortication of the S2AI starting point, it took an average of 4 minutes and 21 seconds (SD 2 minutes 8 seconds, median 4 minutes 15 seconds) from zeroing the IMU-equipped pedicle finder to having the screw fully implanted.

\section{Discussion}

\section{Navigation With IMUs}

Inertial measurement units (IMUs) house accelerometers and gyroscopes to measure acceleration and angular rotation. Inclusion of magnetometers improves the IMUs' accuracy. IMUs are ubiquitous devices: They assist airand spacecraft maneuvering, are used in the safety systems of cars for functions such as airbag deployment and electronic stability control, and detect motion and orientation of tablet computers and smartphones. In this study, the IMUs' data output was processed to track the tilt angles of the IMU-mounted pedicle finder and screwdriver in the axial and sagittal planes.

Our results suggest that S2AI screws can safely and accurately be placed by reproducing preoperatively defined trajectory tilt angles with IMU-equipped surgical tools. Indeed, Kwan et al. measured such angles in a sample of Asian patients. ${ }^{6}$ A surgical tool that is equipped with an IMU enables real-time implementation and monitoring of such angles and may enhance the surgeon's control of instruments. Assessment of the feasibility of this approach to angle-guided spine surgery must address the accuracy of the device; the intraoperative reproducibility of the preoperatively set reference position, timing, and handling of the IMU-equipped tools; the reliability of successful screw placement; and hardware cost. The accuracy of the IMU meets the demands of spine surgery as it is stated to be on the order of $0.5^{\circ}$ to $1^{\circ}$ by the manufacturer (Xsens Technologies) and was confirmed to be within $0.5^{\circ}$ in uniaxial slow motion and within $1^{\circ}$ in multiaxial slow motion (90\%second), ${ }^{7}$ which is within the expected handling velocity of the presented technique to implant S2AI screws.

Over time, the measurements of any IMU may drift away from the initially measured values. Our own accuracy tests with the MTw sensor (Xsens Technologies) showed stable values within a range of $2^{\circ}$ over a time period of at least 4.5 minutes for both angles. This was well

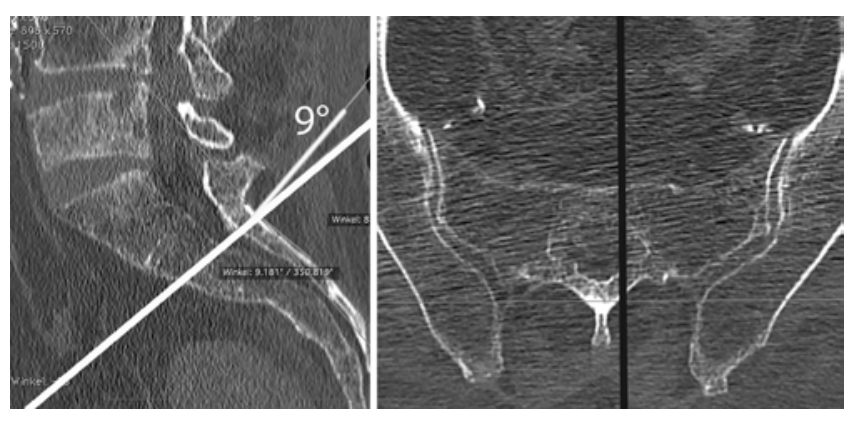

FIG. 2. Multiplanar reconstruction of the preoperative CT scan. The cursor has been shifted from its position in Fig. 1 to the paramedian sacral plane (right panel) while the sagittal projection of the S2AI trajectory (white line in left panel) is maintained. The sagittal tilt is $9^{\circ}$.

within the time range required to cannulate the trajectory or implant the screw.

\section{Zeroing of the Tools}

Zeroing of the IMU was a very quick process, and any edge in the vicinity deemed suitable for preoperative planning of the relative tilt angles and clear intraoperative recognizability could be used for zeroing the IMUs. We chose a plumb line to the paramedian sacral plane as a reference position (Fig. 4 upper). A careful choice for zeroing will ensure optimal final accuracy of the implanted screw, whereas zeroing of the IMUs in a misaligned orientation will inevitably lead to errors in final screw orientation. In other words, deviations of final screw position may be caused by a) inaccurate choice of the starting point, b)
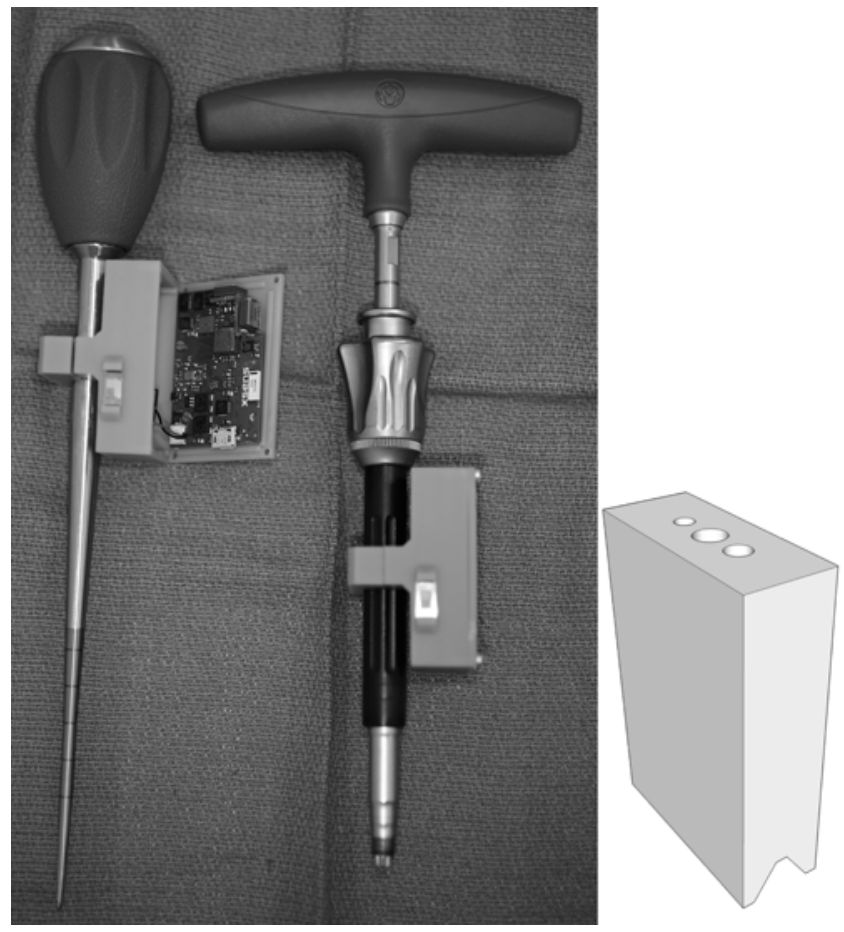

FIG. 3. Left: Inertial measurement units housed in a 3D-printed box are mounted on the pedicle finder and screwdriver. Right: A custom-made 3D-printed box was used for zeroing the pedicle finder and screwdriver in the last 2 cadavers. It has 2 holes in which the pedicle finder or screwdriver can be placed for simplified zeroing. 


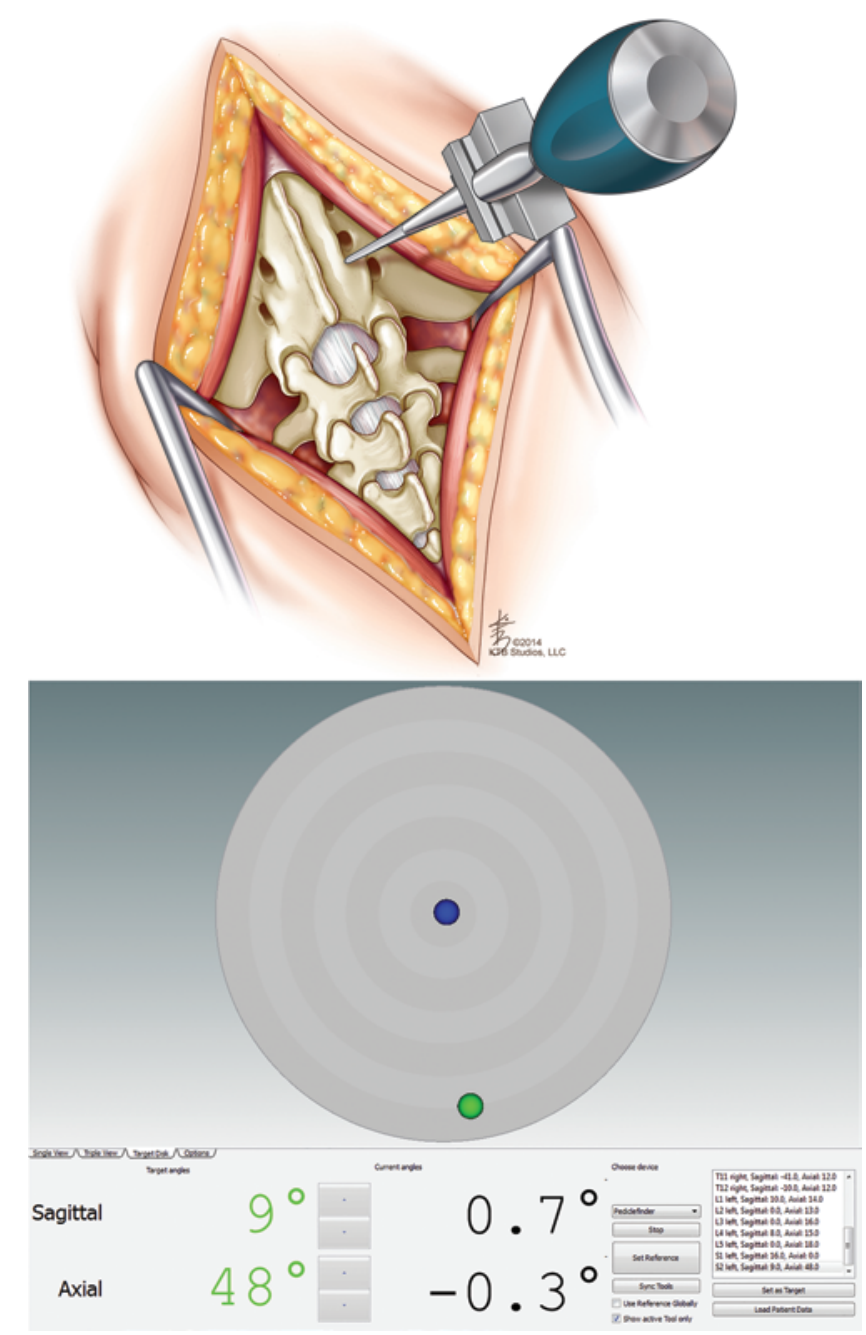

FIG. 4. Zeroing of the pedicle finder. Upper: In the sagittal plane the pedicle finder is aligned perpendicular to the paramedian sacral plane and neutral in the axial plane. ( KTB Studios, 2014. All rights reserved. Published with permission. Lower: On the computer display, the orientation of the IMU-equipped tool (pedicle finder or screwdriver) is represented as blue dot or black digits. Shown here is the quasi-zero or reference position. The green dot and green digits represent the target orientation of the tool.

inaccurate reproduction of the surgeon-controlled intraoperative "zero" orientation of the IMU-equipped tools, c) arbitrarily veering off the IMU-guided trajectory while preparing the canal or implanting the screw, or d) any inaccuracy inherent to the IMU. Despite these potential pitfalls, more than $80 \%$ of the final IMU measurements before releasing the screw were within a $5^{\circ}$ deviation of the postoperative control and $94 \%$ to $100 \%$ were within a deviation of $10^{\circ}$, suggesting that the IMU measurements were reliable (Fig. 8). To facilitate zeroing of the IMUequipped tools, a 3D-printed box with inlets for the pedicle finder and screwdriver was used in Specimens 9 and 10 (Fig. 3 right).

\section{Determining the Starting Point}

Using the same starting point for implanting the S2AI screw as in the preoperative planning is another critical

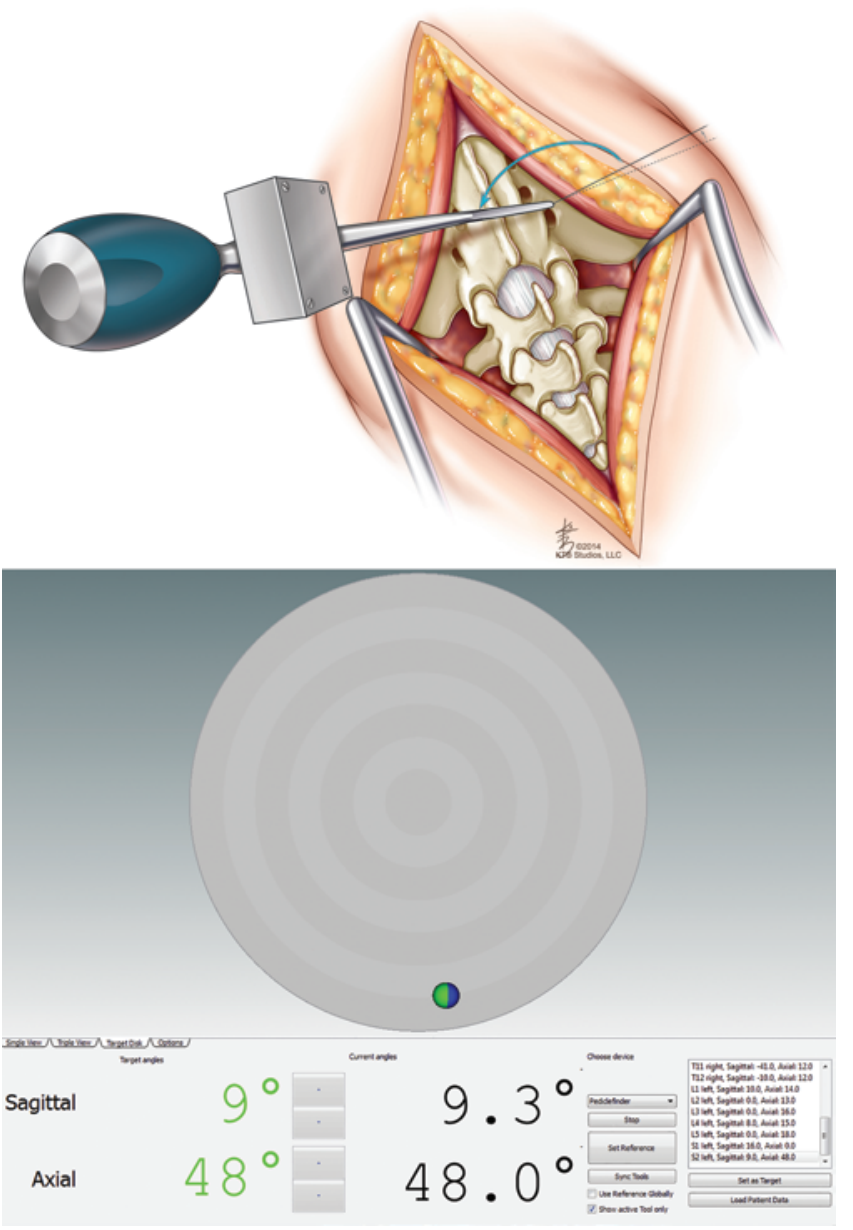

FIG. 5. Cannulation of the S2AI trajectory. Upper: With the tip of the pedicle finder or screwdriver at the starting point for the S2Al screw, the orientation of the pedicle finder or screwdriver is adjusted. (C KTB Studios, 2014. All rights reserved. Published with permission. Lower: With the adjustment, the blue dot (tool) overlaps the green dot (target orientation). The tool is then advanced for cannulation or placement of the S2Al screw.

point for accuracy. The dorsal foramen of S-1 and hence the starting point $1 \mathrm{~mm}$ caudal and lateral to its caudal border can usually be well exposed. Local anatomy was somewhat less clear in Specimen 7: The intraoperative starting point was chosen too far cranially and medially, and strict adherence to the planned tilt angles resulted in a breach into the pelvis. The screws were removed and inserted with a deliberately more divergent axial tilt angle, resulting in a postoperative deviation of approximately $10^{\circ}$. This problem may be avoided by knowing and measuring the distance between an unequivocal landmark, such as the transverse process of L-5, and the starting point for the S2AI screw.

\section{Surgical Experience Using IMUs}

Handling of the IMU-equipped pedicle finder and screwdriver was straightforward. The IMU housing did not interfere with handling of the pedicle finder and screwdriver and did not obscure the view of the tips of the instruments. A benefit of IMU-guided surgery as opposed 


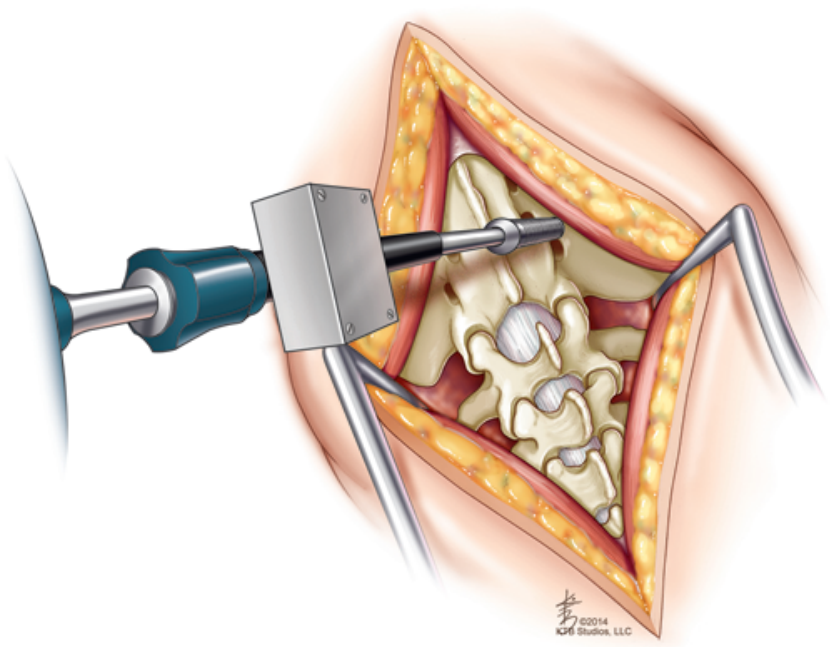

FIG. 6. Placement of the S2AI screw. After the zeroing is repeated on the paramedian sacral plane with the screwdriver (not shown), the tip of the instrument is moved to the starting point and sagittal and axial tilt are adjusted according to the target position on the computer display (as in Fig. 5 lower). ( KTB Studios, 2014. All rights reserved. Published with permission.

to stereoscopic camera-dependent navigation is that an absolute line of sight between the computer and sensor is not required. Of note, our results were achieved without the use of intraoperative fluoroscopy. Although we would not advise against the confirmatory use of intraoperative fluoroscopy in a clinical scenario, e.g., to confirm with an anteroposterior view that the trajectory of the S2AI screw is indeed pointing toward the trochanter major, ${ }^{10}$ the experimental setup of our study allowed us to demonstrate that fluoroscopy is not a sine qua non for IMU-guided implantation of S2AI screws. The results of the presented technique are promising, as 17 of $18 \mathrm{~S} 2 \mathrm{AI}$ screws were correctly placed. In the instance of the single misplaced S2AI screw, the intrapelvic trajectory was indicated by a progressive IMU-monitored offset from the planned angle in the axial plane and hence was noted during the surgery. We deliberately did not attempt to redirect this screw.

\section{Availability of IMUs and Current Medical Use}

Thanks to the ubiquitous use in smartphones and tablet computers, retail prices for IMUs start below US \$100 and thus pose an attractive alternative to much more expensive systems for motion tracking or intraoperative guidance. Indeed, IMUs can be used to monitor trunk, ${ }^{4}$ shoulder and elbow, ${ }^{2}$ and gait $^{3}$ kinematics. In the operating room, IMUenabled techniques are already in clinical use to improve the surgical precision of total knee and hip replacement ${ }^{9}$ and to facilitate percutaneous placement of lumbosacral pedicle screws. ${ }^{5}$ We have also tested the technique for thoracic and lumbar pedicle screws, and final analysis is pending.

\section{Comparing IMU Guidance With 3D Navigation}

Compared with state-of-the-art 3D navigation, the presented IMU-guided navigation is more simple and lightweight. It requires only IMU-equipped tools and a regular
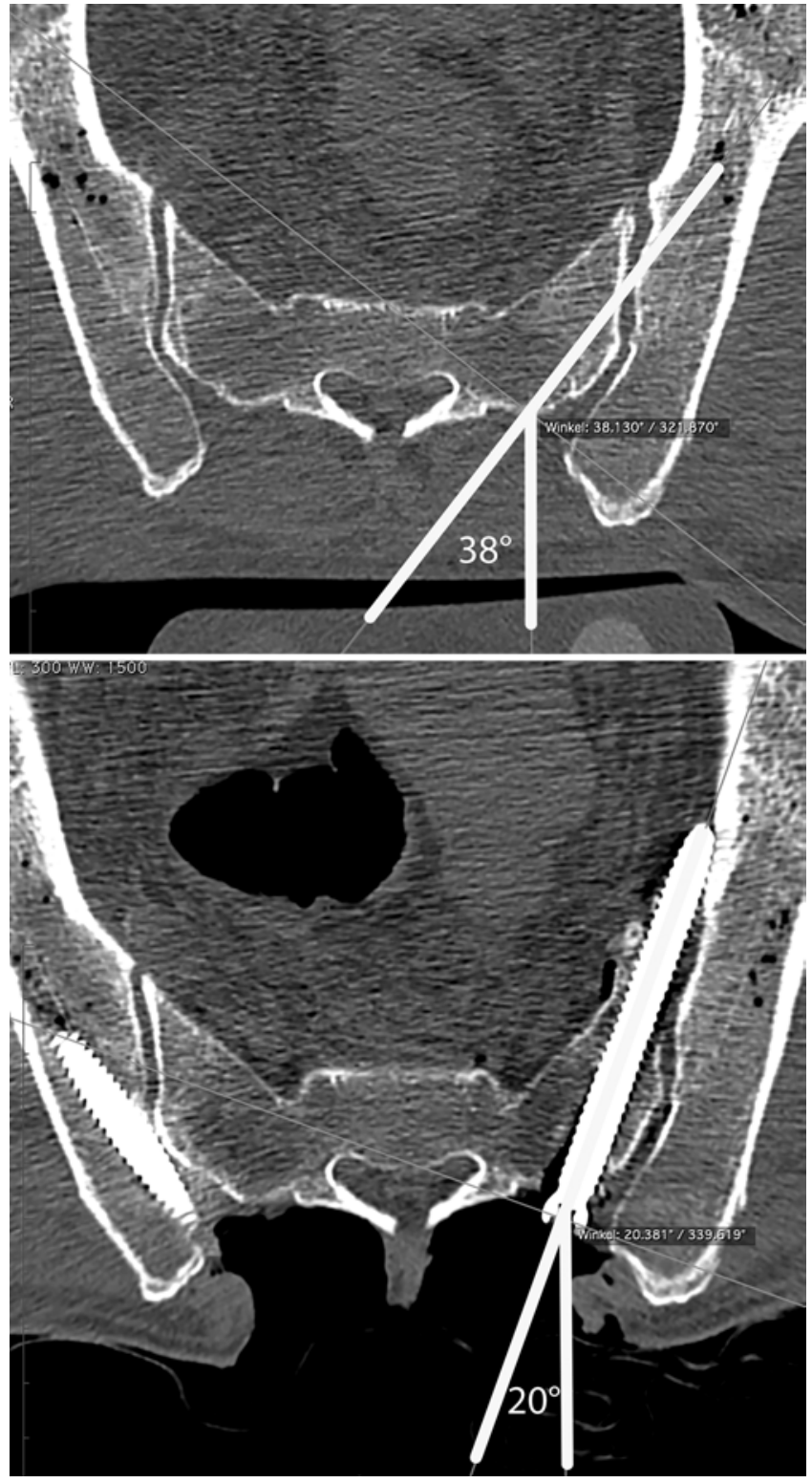

FIG. 7. Axial CT images obtained before and after placement of the screw that breached into the pelvis past the sacroiliac joint. Upper: Planned axial tilt angle of $38^{\circ}$ for the left S2AI screw. Lower: During implantation of the left S2AI screw past the sacroiliac joint, the IMU on the screwdriver indicated a smaller axial tilt angle than planned. This screw was the second one placed in the study, and it was inserted without prior tapping across the SI joint, which we identified as the cause of final misplacement. The final axial offset was $18^{\circ}$.

notebook computer. However, it does not deliver the same amount of information as automated 3D navigation: 1) Whereas 3D navigation can be used to find the ideal trajectory, depth, and starting point, which is visualized on the workstation's screen, the presented IMU-guided technique requires the surgeon to select the starting point for the screw based on thorough knowledge of the exposed intraoperative local anatomy and/or intraoperative fluoroscopy. Therefore, this IMU-guided technique in its current version is not applicable to percutaneous screw placement. 2) Potential errors may occur during the surgeon-controlled 

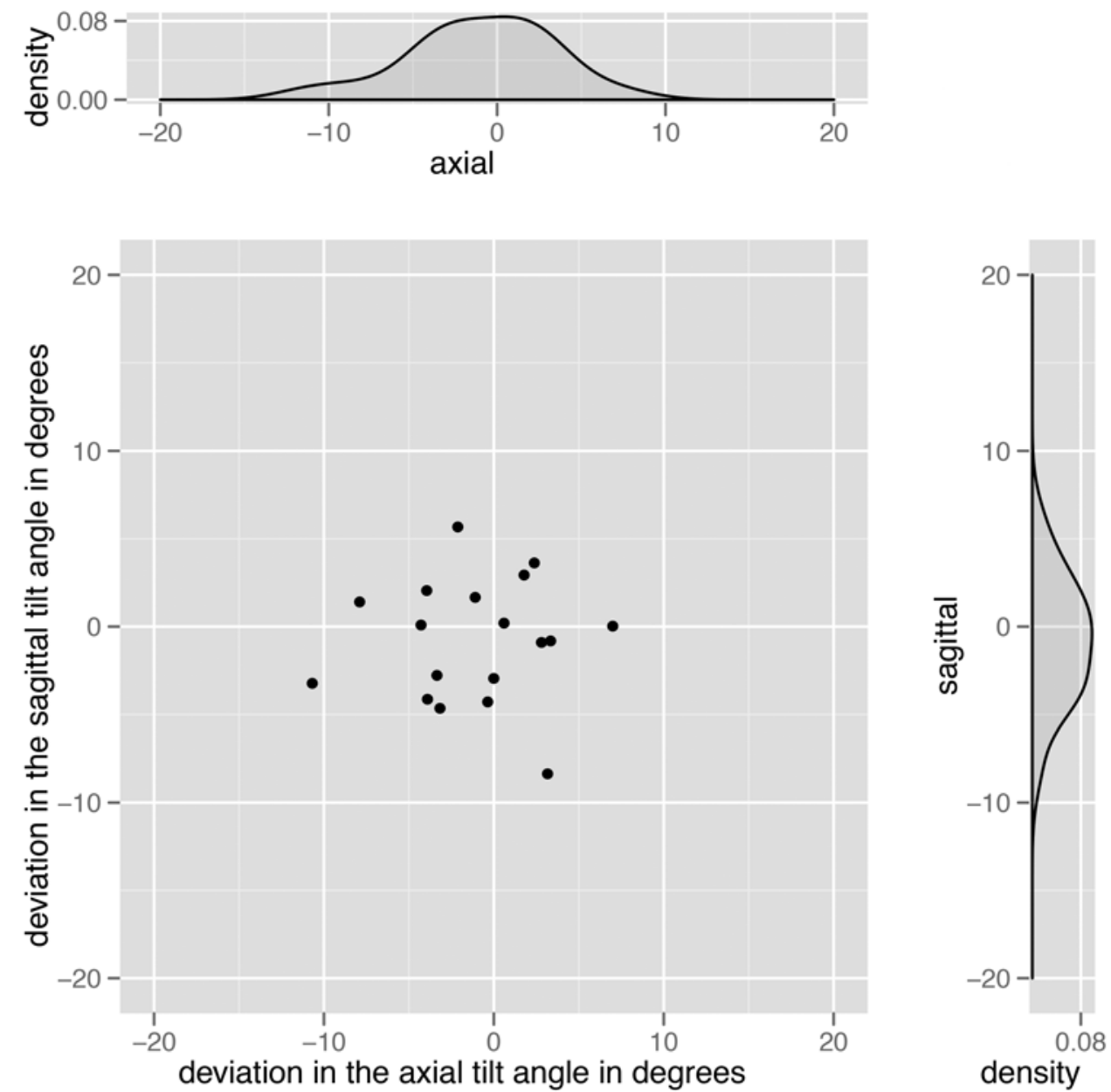

FIG. 8. Scatterplot illustrating the deviation in degrees between IMU measurements and final screw trajectories. Every dot represents an implanted S2AI screw. The deviation between the tilt angle measured by the IMU on the screwdriver and the tilt angle measured on the postoperative CT scan is represented on the x-axis for the axial tilt angle and on the $y$-axis for the sagittal tilt angle. Such deviations may be caused by 1) inaccurately referencing the IMU-equipped screwdriver intraoperatively, 2) inaccurately setting the reference line on the postoperative control CT, or 3) any inaccuracy inherent to the IMU.

planning of the trajectory and, as mentioned above, intraoperative zeroing of the device, whereas errors happen in $3 \mathrm{D}$ navigation if the dynamic reference array is shifted unnoticed after referencing or is attached too far from the point of interest. 3) In contrast to 3D navigation, the depth of advancement is not monitored by the IMU. It must be controlled with the depth readings on the tools, a depth indicator, or fluoroscopy and compared with the surgical plan. 4) The current software version for IMU-guided implantation of S2AI screws requires the surgeon to measure and feed the software with the relative tilt angles ahead of the surgery; however, advanced software using $3 \mathrm{D}$ rendering technology is being developed, and it may eliminate most if not all of the preoperative trajectory planning.

\section{Conclusions}

IMU guidance may offer an inexpensive and convenient tool for assisting with determining angles for placement of S2AI screws for instrumentation in the pelvis. Our results showed that S2AI screws can accurately be implanted by starting $1 \mathrm{~mm}$ caudal and lateral to the caudal border of the dorsal foramen of S-1 and adhering to IMU-guided sagittal and axial tilt angles. Further development of the prototype into a clinically applicable device may assist surgeons in successful placement of S2AI screws.

\section{Acknowledgments}

We wish to thank Magdalena Müller-Gerbl, Nicole Hauser, Sandra Blache, Roger Kurz, and Peter Zimmermann from the Institute of Anatomy, University of Basel, for their kind collaboration. We also thank Philipp Jaszczuk from the Department of Neurosurgery at the University Hospital Basel and Kristin Kraus for editorial assistance.

\section{References}

1. Chang TL, Sponseller PD, Kebaish KM, Fishman EK: Low profile pelvic fixation: anatomic parameters for sacral alariliac fixation versus traditional iliac fixation. Spine (Phila Pa 1976) 34:436-440, 2009

2. Cutti AG, Giovanardi A, Rocchi L, Davalli A, Sacchetti R: Ambulatory measurement of shoulder and elbow kinematics through inertial and magnetic sensors. Med Biol Eng Comput 46:169-178, 2008 
3. Ferrari A, Cutti AG, Garofalo P, Raggi M, Heijboer M, Cappello A, et al: First in vivo assessment of "Outwalk": a novel protocol for clinical gait analysis based on inertial and magnetic sensors. Med Biol Eng Comput 48:1-15, 2010

4. Giansanti D, Maccioni G, Benvenuti F, Macellari V: Inertial measurement units furnish accurate trunk trajectory reconstruction of the sit-to-stand manoeuvre in healthy subjects. Med Biol Eng Comput 45:969-976, 2007

5. Idler C, Rolfe KW, Gorek JE: Accuracy of percutaneous lumbar pedicle screw placement using the oblique or "owl's-eye" view and novel guidance technology. J Neurosurg Spine 13:509-515, 2010

6. Kwan MK, Jeffry A, Chan CYW, Saw LB: A radiological evaluation of the morphometry and safety of S1, S2 and S2ilium screws in the Asian population using three dimensional computed tomography scan: an analysis of 180 pelvis. Surg Radiol Anat 34:217-227, 2012

7. Lebel K, Boissy P, Hamel M, Duval C: Inertial measures of motion for clinical biomechanics: comparative assessment of accuracy under controlled conditions - effect of velocity. PLoS One 8:e79945, 2013

8. Matteini LE, Kebaish KM, Volk WR, Bergin PF, Yu WD, O'Brien JR: An S-2 alar iliac pelvic fixation. Technical note. Neurosurg Focus 28(3):E13, 2010

9. Nam D, Weeks KD, Reinhardt KR, Nawabi DH, Cross MB, Mayman DJ: Accelerometer-based, portable navigation vs imageless, large-console computer-assisted navigation in total knee arthroplasty: a comparison of radiographic results. J Arthroplasty 28:255-261, 2013

10. O'Brien JR, Yu WD, Bhatnagar R, Sponseller P, Kebaish KM: An anatomic study of the S2 iliac technique for lumbopelvic screw placement. Spine (Phila Pa 1976) 34:E439_ E442, 2009

11. O'Brien JR, Yu W, Kaufman BE, Bucklen B, Salloum K, Khalil S, et al: Biomechanical evaluation of S2 alar-iliac screws: effect of length and quad-cortical purchase as compared with iliac fixation. Spine (Phila Pa 1976) 38:E1250 1255,2013

12. Ray WZ, Ravindra VM, Schmidt MH, Dailey AT: Stereotactic navigation with the O-arm for placement of S-2 alar iliac screws in pelvic lumbar fixation. J Neurosurg Spine 18:490-495, 2013
13. Sponseller PD, Zimmerman RM, Ko PS, Pull ter Gunne AF, Mohamed AS, Chang TL, et al: Low profile pelvic fixation with the sacral alar iliac technique in the pediatric population improves results at two-year minimum follow-up. Spine (Phila Pa 1976) 35:1887-1892, 2010

14. Walti J, Jost GF, Cattin PC: A new cost-effective approach to pedicular screw placement, in Linte CA, Yaniv Z, Fallavollita P, et al (eds): Augmented Environments for ComputerAssisted Interventions. Cham, Switzerland: Springer, 2014

\section{Disclosures}

This study was supported by the Gebert Ruef Foundation. The authors report no conflict of interest concerning the materials or methods used in this study or the findings specified in this paper.

\section{Author Contributions}

Conception and design: all authors. Acquisition of data: Jost, Walti, Cattin. Analysis and interpretation of data: Jost, Walti, Cattin. Drafting the article: Jost. Critically revising the article: all authors. Reviewed submitted version of manuscript: all authors. Approved the final version of the manuscript on behalf of all authors: Jost. Statistical analysis: Jost. Administrative/technical/ material support: Mariani, Cattin. Study supervision: Jost, Cattin. Hardware and software development: Walti, Cattin.

\section{Supplemental Information}

Previous Presentations

Parts of the study were presented as a poster at the Joint Meeting of the Swiss Society of Neurosurgery, Society of Clinical Neurophysiology, and Society for Neuropediatrics, Zurich, Switzerland, June 12-13, 2014, and the applied technology was presented at a workshop during the MICCAI conference in Boston, Massachusetts, September 14-18, 2014.

\section{Correspondence}

Gregory F. Jost, Spine Surgery, University Hospital Basel, Spitalstrasse 21,4031 Basel, Switzerland. email: gregoryjost@ icloud.com. 